\title{
INFINITE DIMENSIONAL PARAMETER IDENTIFICATION FOR STOCHASTIC PARABOLIC SYSTEMS
}

\author{
ShinIchi AIHARA \\ Department of Electrical Engineering, Science University of Tokyo, 1-3, Kagurazaka, Shinjuku-ku, Tokyo, 162, Japan \\ Arunabha BAGCHI \\ Faculty of Applied Mathematics, University of Twente, P.O. Box 217, 7500 AE Enschede, The Netherlands \\ Received April 1988 \\ Revised October 1988
}

\begin{abstract}
The infinite dimensional parameter estimation for stochastic heat diffusion equations is considered using the method of sieves. The consistency property is also studied for the long run data.
\end{abstract}

AMS 1980 Subject Classifications: 93C29, 93E12.

Keywords: parameter estimation, inference for stochastic process, stochastic heat diffusion equation.

\section{Introduction}

The identification of infinite dimensional parameters for stochastic distributed parameter systems becomes important in a wide variety of physical phenomena: stochastic control (Balakrishnan, 1975) and large flexible structures (Curtain and Kotelenez, 1987). In contrast with the identification problem for a finite dimensional unknown parameter vector, it may be impossible to construct an algorithm for generating directly an estimate for an infinite dimensional parameter vector. One possible way to construct a practical estimator is to project the infinite dimensional parameter vector to a finite dimensional subspace. For consistency, one must then check whether the finite dimensional estimate converges to the true one as the number of samples and also the dimension of the projected subspace increase without bound.

In this paper, by using the method of sieves, originally proposed by Grenander (1981), an identification algorithm for identifying the unknown diffusion coefficient of a heat equation is proposed, based on a certain log likelihood type cost function. Nguyen en Pham (1982) used this technique for studying the estimation of time-dependent parameters for lumped systems based on independent observations, and recently Bagchi (1985) succeeded in applying this method to the identification problem for a hereditary system with distributed delay. Here we apply the method proposed in Bagchi (1985) to identify spatially dependent parameters for stochastic parabolic systems.

\section{Stochastic parabolic systems}

We set $G \subset \mathbb{R}^{n}$, an open bounded domain with a regular boundary $\left.\Gamma, T=\right] 0, t_{\mathrm{f}}[$, and we work with the following Hilbert spaces: 
$L^{2}(G)$ is the space of square integrable functions on $G$ with the norm $|\cdot|$ and inner product $(\cdot, \cdot)$ ). (We also use $|\cdot|$ as the norm in $\mathbb{R}^{n}$.)

$H^{m}(G)$ is the $m$ th order Sobolev Space, i.e. the space of functions $\phi$ such that

$$
\phi \in L^{2}(G), \quad D^{\alpha} \phi \in L^{2}(G) \quad \forall \alpha,|\alpha| \leqslant m
$$

$\left(\alpha=\left(\alpha_{1}, \ldots, \alpha_{n}\right), \alpha_{i}\right.$ natural numbers, $|\alpha|=\alpha_{1}+\cdots+\alpha_{n}$ and $\left.D^{\alpha} \phi=\left(\partial^{\alpha_{1}+\cdots+\alpha_{n}} / \partial x_{1}^{\alpha_{1}} \cdots \partial x_{n}^{\alpha_{n}}\right) \phi\right)$.

$$
H_{0}^{m}(G)=\left\{\phi \mid \phi \in H^{m}(G), D^{\alpha} \phi=0 \text { and } \Gamma,|\alpha| \leqslant m-1\right\} \text {. }
$$

$H^{-m}(G)$ is the dual of $H_{0}^{m}(G)$.

Define

$$
V=H_{0}^{1}(G) \subset H=L^{2}(G) \subset V^{\prime}=H^{-1}(G)
$$

where we assume that the injection of $V \rightarrow H$ is compact (Adams, 1975, p. 144). Define the linear operator $A\left(a^{0}\right) \in L\left(V ; V^{\prime}\right)$ such that

$$
\left\langle A\left(a^{0}\right) \phi_{1}, \phi_{2}\right\rangle=\sum_{i=1}^{n} \int_{G} a^{0}(x) \frac{\partial \phi_{1}}{\partial x_{i}} \frac{\partial \phi_{2}}{\partial x_{i}} \mathrm{~d} x
$$

where

$$
0<\alpha \leqslant a^{0}(x) \leqslant \beta \quad \forall x \in \bar{G}
$$

and

$$
a^{0} \in C^{2}(G)
$$

From (C2), the operator $A\left(a^{0}\right)$ with range restricted to $H$ defines an unbounded operator which is still denoted by $A\left(a^{0}\right)$ with the domain $D=H_{0}^{1} \cap H^{2}$.

Let $w$ be a $H$-valued Brownian motion process with the incremental covariance $Q \in L_{1}(H ; H)$ where $L_{1}(H ; H)$ denotes the space of trace class operators.

Consider the following stochastic diffusion equations:

$$
(u(t), \phi)+\int_{0}^{t}\left\langle A\left(a^{0}\right) u(s), \phi\right\rangle \mathrm{d} s=\left(u_{0}, \phi\right)+(w(t), \phi) \quad \forall \phi \in V .
$$

Theorem 1 (Bensoussan, 1971, p. 189; Paradoux, 1975, p. 48). Under (C1), (C2) and

$$
u_{0} \in L^{2}(\Omega ; H) \text {, }
$$

there exists a unique solution $u(t)$ of (2.2) such that

$$
u \in L^{2}\left(\Omega ; C(T ; H) \cap L^{2}(T ; V)\right) .
$$

Theorem 2 (Mizohata, 1973, Theorem 5.5, Chapter 5; Tanabe, 1979, Theorem 1.4, Chapter 3). Under (C1) and $(\mathrm{C} 2),-A$ generates strongly continuous, contraction semigroup $T_{t}$ such that

$$
\mathrm{d} T_{t} / \mathrm{d} t=-A T_{t}, \quad T_{0}=I, \quad\left|T_{t} \phi\right|^{2} \leqslant C \mathrm{e}^{-\alpha t}|\phi|^{2} \quad \text { for some } C>0, \alpha>0, \phi \in H .
$$

Define the auto-correlation operator

$$
\Lambda(t, s)=E\{u(t) \otimes u(s)\},
$$

where

$$
\phi_{1} \otimes \phi_{2}=\phi_{1}\left(\phi_{2}, \cdot\right) .
$$


For $\phi \in H$ and noting that $Q=\sum_{i=1}^{\infty} q_{i} e_{1} \otimes e_{i}\left(e_{i}\right.$; orthonomal basis in $\left.H\right)$, we have

$$
\begin{aligned}
E\{u(t)(u(s), \phi)\} & =T_{t} E\left\{u_{0}\left(u_{0}, T_{s}^{*} \phi\right)\right\}+\sum_{i=1}^{\infty} \int_{0}^{t \wedge s} T_{t-\tau} e_{i}\left(e_{i}, T_{s-\tau}^{*} \phi\right) q_{i} \mathrm{~d} \tau \\
& =T_{t} E\left\{u_{0} \otimes u_{0}\right\} T_{s}^{*} \phi+\int_{0}^{t \wedge s} T_{t-\tau} Q T_{t-\tau}^{*} \phi \mathrm{d} \tau
\end{aligned}
$$

so that

$$
\Lambda(t, s)=T_{t} E\left\{u_{0} \otimes u_{0}\right\} T_{s}^{*}+\int_{0}^{t \wedge s} T_{t-\tau} Q T_{s-\tau}^{*} \mathrm{~d} \tau .
$$

We are interested in the stationary system state and, therefore, we choose the initial condition $u_{0}$ to be

$$
\int_{-\infty}^{0} T_{-\tau} \mathrm{d} w(\tau)
$$

The auto-correlation $\Lambda(t, s)$ then becomes

$$
\Lambda(t-s)=\Lambda(t, s)=\int_{-\infty}^{t \wedge s} T_{t-\tau} Q T_{s-\tau}^{*} \mathrm{~d} \tau
$$

\section{Theorem 3.}

$$
\int_{0}^{\infty}|\Lambda(\tau)|_{L_{2}\left(V^{\prime} ; V\right)}^{2} \mathrm{~d} \tau<\infty
$$

where $L_{2}(\cdot ; \cdot)$ denotes the space of Hilbert-Schmidt operators.

This result can be easily established using the property of $T_{t}$. For more details, refer to Aihara and Sunahara (1989).

\section{Parameter estimation procedure}

Noting that the incremental covariance $Q$ of the Brownian motion process $w$ is a trace class operator, it is not clear how a likelihood function may be defined for the infinite dimensional evolution equation (2.2). Hence, instead of introducing the log-likelihood function as the cost, we consider

$$
\begin{aligned}
L(a)= & -\int_{0}^{t_{f}} \sum_{i=1}^{n}\left(a(x) \frac{\partial u(t)}{\partial x_{i}}, \frac{\partial}{\partial x_{i}}(-\Delta)^{-1} \mathrm{~d} u(t)\right) \\
& -\frac{1}{2} \int_{0}^{t_{f}} \sum_{i, j=1}^{n}\left(a(x) \frac{\partial u(t)}{\partial x_{i}}, \frac{\partial}{\partial x_{i}}\left(\frac{\partial}{\partial x_{j}}(-\Delta)^{-1}\right)^{*} a(x) \frac{\partial u(t)}{\partial x_{j}}\right) \mathrm{d} t
\end{aligned}
$$

where

$$
\Delta=\sum_{i=1}^{n} \partial^{2}(\cdot) / \partial x_{i}^{2},
$$

and from Lions $\left(1969\right.$, p. 195), $(-\Delta)^{-1}$ is defined by

$$
(-\Delta)^{-1}=\sum_{k=1}^{\infty}\left(1 / \lambda_{k}\right) \psi_{k} \otimes \psi_{k},
$$


where $\left\{\psi_{i}\right\}$ is a special orthonormal basis in $H$, which satisfies

$$
-\Delta \psi_{k}=\lambda_{k} \psi_{k}, \quad \psi_{k} \in V
$$

and then

$$
\frac{\partial}{\partial x_{i}}\left(\frac{\partial}{\partial x_{j}}(-\Delta)^{-1}\right)^{*}=\sum_{k=1}^{\infty}\left(1 / \lambda_{k}\right) \frac{\partial}{\partial x_{i}} \psi_{k} \otimes \frac{\partial}{\partial x_{j}} \psi_{k} .
$$

Suppose that $\left\{\phi_{i}\right\}$ is an orthonormal basis in $H$ with values in $H^{1}$. We approximate the unknown parameter $a(x)$ by

$$
a^{k}(x)=\sum_{i=1}^{k} a_{i} \phi_{i}(x) .
$$

Using this approximation, the cost functional $L(a)$ defined by (3.1) can be expressed by

$$
\begin{aligned}
L\left(a^{k}\right)= & -\int_{0}^{t_{f}} \sum_{i=1}^{n}\left(a^{k} \frac{\partial u(t)}{\partial x_{i}}, \frac{\partial}{\partial x_{i}}(-\Delta)^{-1} \mathrm{~d} u(t)\right) \\
& -\frac{1}{2} \int_{0}^{t_{f}} \sum_{i, j=1}^{n}\left(a^{k} \frac{\partial u(t)}{\partial x_{i}}, \frac{\partial}{\partial x_{i}}\left(\frac{\partial}{\partial x_{j}}(-\Delta)^{-1}\right)^{*} a^{k} \frac{\partial u(t)}{\partial x_{j}}\right) \mathrm{d} t .
\end{aligned}
$$

The estimate of $a^{0}$ (truc value) is then determined by maximizing (3.4) with respect to $\left\{a_{1}, a_{2}, \ldots, a_{k}\right\}$ and the estimate becomes

$$
\hat{a}_{k}^{t_{\mathrm{f}}}=\sum_{i=1}^{k} \hat{a}_{i}\left(t_{\mathrm{f}}\right) \phi_{i}(x)
$$

where $\hat{a}_{i}\left(t_{\mathrm{f}}\right)$ satisfies, by defining $\hat{a}\left(t_{\mathrm{f}}\right)=\left\{\hat{a}_{1}\left(t_{\mathrm{f}}\right), \hat{a}_{2}\left(t_{\mathrm{f}}\right), \ldots, \hat{a}_{k}\left(t_{\mathrm{f}}\right)\right\}^{\prime}$,

$$
F^{\left(t_{\mathrm{f}}\right)} \hat{a}\left(t_{\mathrm{f}}\right)=g^{\left(t_{\mathrm{f}}\right)}
$$

and where the matrix $F^{\left(t_{f}\right)}$ and vector $g^{\left(t_{f}\right)}$ have components

$$
f_{i j}^{\left(t_{\mathrm{f}}\right)}=\frac{1}{l_{\mathrm{f}}} \int_{0}^{t_{\mathrm{f}}} \sum_{p, m=1}^{n}\left(\phi_{i} \frac{\partial u(t)}{\partial x_{p}}, \frac{\partial}{\partial x_{p}}\left(\frac{\partial}{\partial x_{m}}(-\Delta)^{-1}\right)^{*} \phi_{i} \frac{\partial u(t)}{\partial x_{m}}\right) \mathrm{d} t
$$

and

$$
g_{j}^{\left(t_{\mathrm{f}}\right)}=-\frac{1}{t_{\mathrm{f}}} \int_{0}^{t_{\mathrm{f}}} \sum_{p=1}^{n}\left(\phi_{j} \frac{\partial u(t)}{\partial x_{p}}, \frac{\partial}{\partial x_{p}}(-\Delta)^{-1} \mathrm{~d} u(t)\right),
$$

respectively.

Remark. $(-\Delta)^{-1}$ can be numerically realized by using the finite difference and/or finite element methods. We do not get into this quest: ${ }^{\prime} n$ in the present paper.

\section{Consistency of the estimate}

In order to check the consistency property of the estimate (3.5), the basic equation (3.6) is rewritten as 
follows. First, from (3.8) we have

$$
\begin{aligned}
g_{j}^{\left(t_{f}\right)=}= & \frac{1}{t_{\mathrm{f}}} \int_{0}^{t_{\mathrm{f}}} \sum_{p, m=1}^{n}\left(\phi_{j} \frac{\partial u(t)}{\partial x_{p}}, \frac{\partial}{\partial x_{p}}\left(\frac{\partial}{\partial x_{m}}(-\Delta)^{-1}\right)^{*} a^{0}(x) \frac{\partial u(t)}{\partial x_{m}}\right) \mathrm{d} t \\
& -\frac{1}{t_{\mathrm{f}}} \int_{0}^{t_{\mathrm{f}}} \sum_{p=1}^{n}\left(\phi_{j} \frac{\partial u(t)}{\partial x_{p}}, \frac{\partial}{\partial x_{p}}(-\Delta)^{-1} \mathrm{~d} w(t)\right) \\
= & \sum_{q=1}^{k} f_{j q}^{\left(t_{\mathrm{f}}\right)} a_{q}^{0}+\frac{1}{t_{\mathrm{f}}} \int_{0}^{t_{\mathrm{f}}} \sum_{p, m=1}^{n}\left(\phi_{j} \frac{\partial u(t)}{\partial x_{p}}, \frac{\partial}{\partial x_{p}}\left(\frac{\partial}{\partial x_{m}}(-\Delta)^{-1}\right)^{*}\right. \\
& \left.-\frac{1}{t_{\mathrm{f}}} \int_{0}^{t_{\mathrm{f}}} \sum_{p=1}^{n}\left(a^{0}(x)-\sum_{q=1}^{k} a_{q}^{0} \phi_{q}(x)\right) \frac{\partial u(t)}{\partial x_{p}}, \frac{\partial}{\partial x_{p}}(-\Delta)^{-1} \mathrm{~d} w(t)\right)
\end{aligned}
$$

where

$$
a_{q}^{0}=\left(a^{0}, \phi_{q}\right)
$$

Hence, setting $h_{j}^{\left(t_{\mathrm{f}}\right)}$ as the 2 nd and $3 \mathrm{rd}$ terms of the right hand side of (4.1), (3.6) becomes

$$
F^{\left(t_{\mathrm{f}}\right)}\left(\hat{a}_{k}\left(t_{\mathrm{f}}\right)-a^{0}(k)\right)=h^{\left(t_{\mathrm{f}}\right)}
$$

where

$$
a^{0}(k)=\left\{a_{1}^{0}, a_{2}^{0}, \ldots, a_{k}^{0}\right\}^{\prime}
$$

and $h_{j}^{\left(t_{f}\right)}$ denotes the $j$ th component of $h^{\left(t_{f}\right)}$.

Lemma 1. $\exists C_{1}>0$ (independent of $i$ and $j$ ) such that

$$
E\left\{\left|f_{i j}^{\left(t_{\mathrm{f}}\right)}-E\left\{f_{i j}^{\left(t_{\mathrm{f}}\right)}\right\}\right|^{2}\right\} \leqslant C_{1} / \mathrm{r}_{\mathrm{f}} .
$$

Proof. From (3.7), it is easy to show that

$$
E\left\{\left|f_{i j}^{\left(t_{\mathrm{f}}\right)}-E\left\{f_{i j}^{\left(t_{\mathrm{f}}\right)}\right\}\right|^{2}\right\}=\frac{1}{t_{\mathrm{f}}^{2}} \int_{0}^{t_{\mathrm{f}}} \int_{0}^{t_{\mathrm{f}}} E\left\{X_{i j}(t) X_{i j}(s)\right\} \mathrm{d} t \mathrm{~d} s
$$

where

$$
\begin{aligned}
X_{i j}(t)= & \sum_{p, m=1}^{n}\left(\phi_{i} \frac{\partial u(t)}{\partial x_{p}}, \frac{\partial}{\partial x_{p}}\left(\frac{\partial}{\partial x_{m}}(-\Delta)^{-1}\right)^{*} \phi_{j} \frac{\partial u(t)}{\partial x_{m}}\right) \\
& -E\left\{\sum_{p, m=1}^{n}\left(\phi_{i} \frac{\partial u(t)}{\partial x_{p}}, \frac{\partial}{\partial x_{p}}\left(\frac{\partial}{\partial x_{m}}(-\Delta)^{-1}\right)^{*} \phi_{j} \frac{\partial u(t)}{\partial x_{m}}\right)\right\}
\end{aligned}
$$


Noting that

$$
\begin{aligned}
E\left\{X_{i j}(t) X_{i j}(s)\right\}= & E\left\{\sum_{p, m=1}^{n}\left(\phi_{i} \frac{\partial u(t)}{\partial x_{p}}, \frac{\partial}{\partial x_{p}}\left(\frac{\partial}{\partial x_{m}}(-\Delta)^{-1}\right)^{*} \phi_{j} \frac{\partial u(t)}{\partial x_{m}}\right)\right. \\
& \left.\times \sum_{p, m=1}^{n}\left(\phi_{i} \frac{\partial u(s)}{\partial x_{p}}, \frac{\partial}{\partial x_{p}}\left(\frac{\partial}{\partial x_{m}}(-\Delta)^{-1}\right)^{*} \phi_{j} \frac{\partial u(s)}{\partial x_{m}}\right)\right\} \\
- & E\left\{\sum_{p, m=1}^{n}\left(\phi_{i} \frac{\partial u(t)}{\partial x_{p}}, \frac{\partial}{\partial x_{p}}\left(\frac{\partial}{\partial x_{m}}(-\Delta)^{-1}\right)^{*} \phi_{j} \frac{\partial u(t)}{\partial x_{m}}\right)\right\} \\
& \times E\left\{\sum_{p, m=1}^{n}\left(\phi_{i} \frac{\partial u(s)}{\partial x_{p}}, \frac{\partial}{\partial x_{p}}\left(\frac{\partial}{\partial x_{m}}(-\Delta)^{-1}\right)^{*} \phi_{j} \frac{\partial u(s)}{\partial x_{m}}\right)\right\}
\end{aligned}
$$

and using the Gaussian property of the state $u$, we get

$$
\begin{gathered}
E\left\{X_{i j}(t) X_{i, j}(s)\right\}=2 \sum_{p, m=1}^{n} \sum_{q, r=1}^{n} E\left\{\left(\phi_{i} \frac{\partial u(t)}{\partial x_{p}}, E\left\{\frac{\partial}{\partial x_{p}}\left(\frac{\partial}{\partial x_{m}}(-\Delta)^{-1}\right)^{*} \phi_{j} \frac{\partial u(t)}{\partial x_{m}} \otimes \phi_{i} \frac{\partial u(s)}{\partial x_{q}}\right\}\right.\right. \\
\left.\left.\times \frac{\partial}{\partial x_{q}}\left(\frac{\partial}{\partial x_{r}}(-\Delta)^{-1}\right)^{*} \phi_{j} \frac{\partial u(s)}{\partial x_{r}}\right)\right\} \\
=2 \sum_{p, m=1}^{n} \sum_{q, r=1}^{n}\left[\phi_{i} \frac{\partial}{\partial x_{p}}\left(\frac{\partial}{\partial x_{q}}\left(\frac{\partial}{\partial x_{r}}(-\Delta)^{-1}\right)^{*} \phi_{j} \frac{\partial \Lambda(t, s)}{\partial x_{r}}\right)^{*},\right. \\
\left.\left(\frac{\partial}{\partial x_{p}}\left(\frac{\partial}{\partial x_{m}}(-\Delta)^{-1}\right)^{*} \phi_{j} \frac{\partial}{\partial x_{m}}\left(\phi_{i} \frac{\partial \Lambda(t, s)}{\partial x_{q}}\right)^{*}\right)\right],
\end{gathered}
$$

where $[\cdot, \cdot]$ denotes the Hilbert-Schmidt inner product. From $(3.2 \mathrm{~d})$, we have

$$
\begin{aligned}
& \left|\frac{\partial}{\partial x_{p}}\left(\frac{\partial}{\partial x_{m}}(-\Delta)^{-1}\right)^{*} \xi\right|^{2} \\
& \quad \leqslant \sum_{p, m=1}^{n} \sum_{k, l=1}^{\infty} \frac{1}{\lambda_{k} \lambda_{l}}\left(\frac{\partial \psi_{k}}{\partial x_{p}}, \frac{\partial \psi_{l}}{\partial x_{p}}\right)\left(\frac{\partial \psi_{k}}{\partial x_{m}}, \xi\right)\left(\frac{\partial \psi_{l}}{\partial x_{m}}, \xi\right) \\
& \quad=\sum_{m=1}^{n} \sum_{k=1}^{\infty} \frac{1}{\lambda_{k}}\left(\frac{\partial \psi_{k}}{\partial x_{m}}, \xi\right)^{2} \quad(\text { from }(3.2 \mathrm{c})) \\
& \quad=\sum_{m=1}^{n} \sum_{k=1}^{\infty} \frac{1}{\lambda_{k}}\left(\psi_{k}, \frac{\partial \xi}{\partial x_{m}}\right)^{2} \quad \text { (from Green's formula) } \\
& \quad=\sum_{m=1}^{n}\left(\frac{\partial \xi}{\partial x_{m}},(-\Delta)^{-1} \frac{\partial \xi}{\partial x_{m}}\right) \quad \text { (from (3.2b)) } \\
& \quad=\sum_{m=1}^{n}\left(\frac{\partial \xi}{\partial x_{m}}, \frac{\partial \xi}{\partial x_{m}}\right)_{H^{-1}} \quad \text { (from Lions, 1969, p. 192) } \\
& \quad=\sum_{m=1}^{n}\left|\frac{\partial \xi}{\partial x_{m}}\right|_{H^{-1}}^{2} \\
& \quad \leqslant C|\xi|^{2}\left(\text { from Lions and Magenes, 1972, p. 85, } \partial / \partial x_{i} \in \mathscr{L}\left(H ; H^{-1}\right)\right)
\end{aligned}
$$


and from the fact that $\phi_{i}$ is an orthonomal basis in $H$,

$$
\left|\phi_{i}\right|^{2}=1
$$

we have

$$
E\left\{X_{i j}(t) X_{i j}(s)\right\} \leqslant \text { Const. }|\Lambda(t, s)|_{L_{2}\left(V^{\prime} ; V\right)}^{2} .
$$

Now, we get

$$
\begin{aligned}
E\left\{\left|f_{i j}^{\left(t_{\mathrm{f}}\right)}-E\left\{f_{i j}^{\left(t_{\mathrm{f}}\right)}\right\}\right|^{2}\right\} & \leqslant \frac{\text { Const. }}{t_{\mathrm{f}}^{2}} \int_{0}^{t_{\mathrm{f}}} \int_{0}^{t_{\mathrm{f}}}|\Lambda(t, s)|_{L_{2}\left(V^{\prime}: V\right)}^{2} \mathrm{~d} t \mathrm{~d} s \\
& \leqslant \frac{\text { Const. }}{t_{\mathrm{f}}}\left(\int_{0}^{t_{\mathrm{f}}}|\Lambda(\tau)|_{L_{2}\left(V^{\prime} ; V\right)}^{2} \mathrm{~d} \tau+\int_{-t_{\mathrm{f}}}^{0}|\Lambda(\tau)|_{L_{2}\left(V^{\prime}: V\right)}^{2} \mathrm{~d} \tau\right) \\
& \leqslant \frac{\text { Const. }}{t_{\mathrm{f}}}\left(\int_{-\infty}^{\infty}|\Lambda(\tau)|_{L_{2}\left(V^{\prime}: V\right)}^{2} \mathrm{~d} \tau\right) \\
& \leqslant \text { Const. } / t_{\mathrm{f}} .
\end{aligned}
$$

\section{Lemma 2.}

$$
\begin{aligned}
\lim _{t_{\mathrm{f}} \rightarrow \infty} f_{i j}^{\left(t_{\mathrm{f}}\right)} & =\sum_{p, m=1}^{n} \operatorname{tr}\left\{\phi_{i} \frac{\partial}{\partial x_{p}}\left(\frac{\partial}{\partial x_{p}}\left(\frac{\partial}{\partial x_{m}}(-\Delta)^{-1}\right)^{*} \phi_{j} \frac{\partial}{\partial x_{m}} \Lambda(0)\right)^{*}\right\} \\
& =\sum_{p, m=1}^{n}\left[\frac{\partial}{\partial x_{p}}\left(\frac{\partial}{\partial x_{m}}(-\Delta)^{-1}\right)^{*}, \phi_{i} \frac{\partial}{\partial x_{p}}\left(\phi_{j} \frac{\partial}{\partial x_{m}} \Lambda(0)\right)^{*}\right] \text { a.s. }
\end{aligned}
$$

The proof of this lemma can be easily derived from Theorem 3 and Gaussian property of $u$. For more details, refer to Aihara and Sunahara (1989) and Wong (1971, p. 69).

Lemma 3. $\exists C_{3}>0$ (independent of $j$ and $t_{\mathrm{f}}$ ),

$$
E\left\{\left|h_{j}^{\left(t_{\mathrm{f}}\right)}\right|^{2}\right\} \leqslant C_{3}\left(1 / t_{\mathrm{f}}+\left|I_{j}(k)\right|^{2}\right)
$$

where $I_{j}(k)$ is given by (4.17) below and

$$
\sum_{j=1}^{\infty}\left|I_{j}(k)\right|^{2} \rightarrow 0, \quad \text { as } k \rightarrow \infty .
$$

Proof. We set

$$
I_{j}(k)=\frac{1}{t_{\mathrm{f}}} E\left\{\int_{0}^{t_{f}} \sum_{p, m=1}^{n}\left(\phi_{j} \frac{\partial u(t)}{\partial x_{p}}, \frac{\partial}{\partial x_{p}}\left(\frac{\partial}{\partial x_{m}}(-\Delta)^{-1}\right)^{*}\left(a^{0}(x)-\sum_{q=1}^{k} a_{q} \phi_{q}(x)\right) \frac{\partial u(t)}{\partial x_{m}}\right) \mathrm{d} t\right\}
$$


It is easy to show that from Lemma 2,

$$
\begin{aligned}
\sum_{j=1}^{\infty}\left|I_{j}(k)\right|^{2} & \leqslant\left\{\frac{\text { Const. }}{t_{\mathrm{f}}} E\left\{\int_{0}^{t_{\mathrm{f}}}|u(t)|_{v}^{2} \mathrm{~d} t\right\}\left|a^{0}-\sum_{q=1}^{k} a_{q}^{0} \phi_{q}\right|\right\}^{2} \\
& \leqslant \text { Const. }|\Lambda(0)|_{L_{1}\left(V^{\prime} ; V\right)}^{2}\left|a^{0}-\sum_{q=1}^{k} a_{q}^{0} \phi_{q}\right|^{2} \rightarrow 0, \text { as } k \rightarrow \infty
\end{aligned}
$$

From (4.1), we have

$$
\begin{aligned}
& E\left\{\left|h_{j}^{\left(t_{f}\right)}\right|^{2}\right\} \\
& \leqslant 3 E\left\{\frac{1}{t_{\mathrm{f}}^{2}} \int_{0}^{t_{\mathrm{f}}} \int_{0}^{t_{\mathrm{f}}}\left\{\sum_{p, m=1}^{n}\left(\phi_{j} \frac{\partial u(t)}{\partial x_{p}}, \frac{\partial}{\partial x_{p}}\left(\frac{\partial}{\partial x_{m}}(-\Delta)^{-1}\right)^{*} \sum_{q=k+1}^{\infty} a_{q}^{0} \phi_{q} \frac{\partial u(t)}{\partial x_{m}}\right)-I_{j}(k)\right\}\right. \\
& \left.\times\left\{\sum_{p, m=1}^{n}\left(\phi_{j} \frac{\partial u(t)}{\partial x_{p}}, \frac{\partial}{\partial x_{p}}\left(\frac{\partial}{\partial x_{m}}(-\Delta)^{-1}\right)^{*} \sum_{q=k+1}^{\infty} a_{q}^{0} \phi_{q} \frac{\partial u(s)}{\partial x_{m}}\right)-I_{j}(k)\right\} \mathrm{d} s \mathrm{~d} t\right\} \\
& +3 E\left\{\frac{1}{t_{\mathrm{f}}^{2}}\left|\int_{0}^{t_{\mathrm{f}}} \sum_{p=1}^{n}\left(\phi_{i} \frac{\partial u(t)}{\partial x_{p}}, \frac{\partial}{\partial x_{p}}(-\Delta)^{-1} \mathrm{~d} w(t)\right)\right|^{2}\right\}+3\left|I_{j}(k)\right|^{2} .
\end{aligned}
$$

By using the same procedure to derive the results of Lemma 1, we can show that there exists a $C>0$ such that

the 1st term of the right hand side of $(4.19) \leqslant C / t_{\mathrm{f}}$.

Furthermore, it follows that

$$
\begin{aligned}
& E\left\{\frac{1}{t_{\mathrm{f}}^{2}}\left|\int_{0}^{t_{f}} \sum_{p=1}^{n}\left(\phi_{j} \frac{\partial u(t)}{\partial x_{p}}, \frac{\partial}{\partial x_{p}}(-\Delta)^{-1} \mathrm{~d} w(t)\right)\right|^{2}\right\} \\
& \quad=\frac{1}{t_{\mathrm{f}}^{2}} E\left\{\int_{0}^{t_{\mathrm{f}}} \sum_{m, p=1}^{n}\left(\phi_{j} \frac{\partial u(t)}{\partial x_{p}}, \frac{\partial}{\partial x_{p}}(-\Delta)^{-1}\left(\frac{\partial}{\partial x_{m}}(-\Delta)^{-1} Q\right)^{*} \phi_{j} \frac{\partial u(t)}{\partial x_{m}}\right) \mathrm{d} t\right\} \\
& \quad \leqslant\left(C / t_{\mathrm{f}}\right)|\Lambda(0)|_{L_{1}\left(V^{\prime} ; V\right)}
\end{aligned}
$$

Theorem 4. If $k \rightarrow \infty$, with $k / t_{\mathrm{f}} \rightarrow 0$, as $t_{\mathrm{f}} \rightarrow \infty$, then

$$
\lim _{t_{\mathrm{r}} \rightarrow \infty} \sum_{p, m=1}^{n}\left|\frac{\partial}{\partial x_{p}}\left(\frac{\partial}{\partial x_{p}}\left(\frac{\partial}{\partial x_{m}}(-\Delta)^{-1}\right)^{*}\left(\left(\hat{a}_{k}^{t_{r}}-a^{0}\right) \frac{\partial \Lambda(0)}{\partial x_{m}}\right)^{*}\right)\right|_{L_{2}(H ; H)}=0
$$

in probability. 
Proof. From Lemma 3, it follows that

$$
\begin{aligned}
E\left\{\left|F^{\left(t_{\mathrm{f}}\right)}\left(\hat{a}_{k}^{t_{\mathrm{f}}}-a^{0}(k)\right)\right|^{2}\right\} & =\sum_{j=1}^{k} E\left\{\left|h_{j}^{\left(t_{\mathrm{f}}\right)}\right|^{2}\right\} \\
& \leqslant \text { Const. }\left(\frac{k}{t_{\mathrm{f}}}+\sum_{j=1}^{\infty}\left|I_{j}(k)\right|^{2}\right) \rightarrow 0, \text { as } k, t_{\mathrm{f}} \rightarrow \infty .
\end{aligned}
$$

Using Lemma 2, we can derive (4.22) from (4.23).

\section{Conclusion}

We studied the identification problem for parabolic systems with spatially dependent parameter. Our state was assumed to be completely observed. The problem becomes more complicated for the case of partial observation. For the case of finite dimensional parameter vectors, this problem has been analyzed in Bagchi and Borkar (1984). The extension to infinite dimensional parameter vectors is not obvious at present.

\section{References}

Adams, R.A. (1975), Sobolev Spaces (Academic Press, New York).

Aihara, S.I. and Y. Sunahara (1988), Identification of an infinite dimensional parameter for stochastic diffusion equations, SIAM J. Control Optim. 26, 1062-1075.

Bagchi, A. (1985), Identification for a hereditary system with distributed delay, System Control Lett. 5, 339-345.

Bagchi, A. and V. Borkar (1984), Parameter identification on infinite dimensional linear systems, Stochastics 12, 201-213.

Balakrishnan, (1975), Identification and stochastic control of a class of distributed parameter systems with boundary noise, in: Lecture Notes in Econom. Math. Systems, Vol. 107 (Springer, New York).

Bensoussan, A. (1971), Filtrage Optimal des System Lineaires (Dunod, Paris).

Curtain, R.F. and P. Kotelenez (1987), Stochastic model for uncertain flexible systems, Automatica 23, 657-661.
Grenander, U. (1981), Abstract Inference (Wiley, New York). Lions, J.L. (1969), Quelques Methodes de Resolution des Problemes aux Limites Non Lineaires (Dunod, Paris).

Lions, J.L. and E. Magenes (1972), Non-Homogeneous Boundary Value Problems and Applications, Vol. I (Springer, Berlin).

Mizohata, S. (1973), The Theory of Partial Differential Equations (Cambridge Univ. Press, Cambridge).

Nguyen, H. and T.D. Pham (1982), Identification of nonstationary model by the method of sieves, SIAM J. Control Optim. 20 603-611.

Paradoux, E. (1975), Equations aux derivees partielles stochastiques non lineaireș monotones, Thesis, Université Paris XI (Paris).

Tanabe, H. (1979), Equations of Evolutions (Pitman, London). Wong, E. (1971), Stochastic Processes in Information and Dynamical Systems (McGraw-Hill, New York). 